\title{
Understanding the Effects of Cognition in Creative Decision Making: A Creativity Model for Enhancing the Design Studio Process
}

\author{
Deniz Hasirci and Halime Demirkan \\ Bilkent University
}

\begin{abstract}
The essential components of creativity - persons, processes and products - were investigated inside a creative environment by deeply focusing on the cognitive stages of the creative decision making process. Mental imagery and external representation were considered as the implicit parts of creativity for enhancing design studio process. An experiment was conducted with 15 subjects who designed the public area of a train as the task in design studio. Observation, protocol analysis, and rating scales were used as assessment tools. Considering the components of creativity, it was found that the highest correlation was between process and overall creativity. Person and product followed process, respectively. However, no significant relationship was observed between imagery and creativity in design process. Three-dimensional representations were found to lead to more creativity compared to 2-dimensional depictions
\end{abstract}

Various attempts have been made to understand creativity in different design fields. Creativity as a natural component of design process has, for example, often been characterized by the occurrence of an event called the creative leap. Cross (1997) explained the creative leap as a bridge between the problem space and solution space by the identification of a key concept in design process. In other attempts, the works of successful architects (Lawson, 1994) or product designers (Candy \& Edmonds, 1996; Cross, 2002; Roy \& Design Innovation Group, 1993) have been examined in order to discover the relevant creative personal traits. However, Lloyd and Snelders (2003) argued that "a designer's personal creativity does form a necessary condition for a design's success, but it will never be a sufficient condition" (p. 252). Christiaans (1992) studied design students with a particular intention of looking at creativity in industrial design.

Attempts to understand and promote creative thinking have focused on a number of descriptive models. Rosenman and Gero (1993), for example, classified the procedures that might occur in creative design models into four groups: combination, mutation, analogy, and first principles. Gero (1994) added emergence to these groups. Hennessey (1994) focused on the assessment of creativity by examining the relationship between ratings of product and process creativity. Dorst and Cross (2001) also studied this relationship, and using protocol analysis in their empirical study, evaluated the observations in a model of creative design as the coevolution of the problem and solutions spaces. They claimed that the process of evolution, as driven by a reaction to surprise, could be considered as creativity in the design process. Krueger and Cross (2001) studied nine experienced industrial designers to develop an expertise model of product design process. They identified four groups of driven design strategies as problem, information, solution, and knowledge, and evaluated the outcomes in terms of solution quality and creativity. They found that designers using a problem-driven strategy produced the best results

Correspondence should be sent to Deniz Hasirci, Faculty of Art, Design, and Architecture, Department of Interior Architecture and Environmental Design, Bilkent University, 06800 Bilkent, Ankara, Turkey. E-mail: hasirci@bilkent.edu.tr 
in terms of balance of both overall solution quality and creativity. A high creativity score was obtained in both solution and knowledge driven design strategies.

In architectural design processes, Akin and Akin (1996) analyzed the discovery of a creative solution that corresponds to the sudden attainment of an insight (A ha!) in the sketch of a design problem that was structured with several restricting frames of reference. Akin and Akin (1998) later suggested that "the cognitive processes observed in these design fields closely resemble processes that play a role in a number of the traditional art fields such as music, writing, painting and sculpture" (pp. 129-130). This was supported by Christiaans' (2002) study, in which he found that the judgments by people with different levels of design expertise do not differ from art assessment ones. He also concluded that there was no difference in the judgment of experts and nonexperts of design field.

Rhodes (1961/1987) classified the creative studies into four categories: the creative person (the person who creates), the process (the process of creation), the product (the product that is a result of the creative process), and the press or environment (environment, context, or situation in which the creative act takes place). It was abbreviated as 4P's. Runco (2004) reviewed creativity research in the past 20 years under the headings of person, product, process, and press, and concluded that there is a recent increase in interdisciplinary effort in scientific creativity research. Among all sorts of investigations regarding creativity, there is a lack of studies that investigate the stages within the creative process - one constituent of the 4P's. Additionally, there are not many studies in design settings that examine the creativity-setting relationship (situations or conditions that enhance and promote creative decision-making).

Creativity can only become recognizable if there exists an interrelation of the 4P's (Isaksen, Murdock, Firestien \& Treffinger, 1993; Jones, 1993; Mooney, 1963; Runco, 2004). Simonton (2003) stated that creativity has three essential components-persons, processes, and products - and they should all be investigated for a complete notion of the concept, otherwise, instead of the "forest" of creativity one can only see "singular trees" without the picture of the whole (p. 490). Hasirci and Demirkan
(2003) focused on the interaction between the creative person, creative process, and creative product inside a creative environment during a design process. This study considers these elements but delves deeper into the creative process. It analyzes the cognitive stages of creative decision-making process during the act of designing. The creative environment that was used as a variable in the previous study of the same authors (Hasirci \& Demirkan, 2003) is now used as a constant, and its physical and social features are investigated. All of the four components are considered since creativity is not a personality trait that is separate from everything else, but a total assessment of the individual by the social system in terms of "patterns of traits that are characteristic of creative persons" (Guilford, 1968, p. 78). Understanding the processes within these components, in turn, leads to the activity necessary to support them, and the totality of creativity. Therefore, the cognitive stages of creative problem solving should be methodically investigated.

\section{Cognitive Stages of Creative Problem-Solving}

Design, a field that inherently involves a creative problem-solving activity, necessitates the making of decisions in order to fulfil certain objectives. Over the years, several different models have been proposed to explain the process of creative problem solving. In fact, these models are not extremely different from each other and have quite a lot in common. The first of these models was originated by Wallas (1926) and consists of four stages: preparation, incubation, illumination, and verification. Plsek (1997) claimed that, in establishing the stages of creative process, many models use the common theme (such as Bandrowski's, 1985 Model for Creative Strategic Planning; Barron's, 1988, Psychic Creation Model; Fritz's, 1991, Process for Creation; Isaksen \& Treffinger's, 1985, Creative Problem-Solving Model; Koberg \& Bagnall's, 1981, Universal Traveler Model; Osborn's, 1953, Seven Step Model for Creative Thinking; Rossman's, 1931, Creativity Model). A model proposed by Jones (1992) that explains the same process for the field of design has similar stages. 
The five stages (5R's) of the Sensational Thinking model of O'Neill and Shallcross (1994) separates itself from other models as it describes perception as a naturally occurring dynamic system within the creative process. This is especially important because it helps to link cognition and creativity by enabling the relation between the 5R's Model of sensational thinking, and the 4P's model of creativity that embodies the creative process of decision making with an emphasis on its stages. In each stage, idea establishing and the nature of how this is done are significant. The characteristics of the Sensational Thinking model are closely related to the dynamic nature of design process. The 5R's are adapted to the context of design literature as follows:

1. Readiness: Relaxation activity that necessitates letting go and being open to the possibilities. Preparation, analysis, and initial idea conception can be seen at this stage. Involves more imagery than representation, as well as looking around for ideas and observing the task intently.

2. Reception: To experience fully and observe with all the senses. Imagination, generation, idea selection, or refinement is evident at this stage. Shifting and redefining the problem space which is a critical aspect of creativity takes place (Akin \& Akin, 1998). When the person passes from observing the task to externalizing the initial ideas, reflection stage has begun.

3. Reflection: Remembering activity and allowing time for internal interaction. It is a stage at which harvesting, evaluation, idea development, enriching and expanding discovery takes place to prepare the person for the next step. $\mathrm{He}$ or she may alternate between rigorous sessions of imagery and representation at this stage, and when one of the options is chosen among alternatives, the next stage has begun (Akin \& Akin, 1998; Jones, 1992; Kristensen, 2004; O’Neill \& Shallcross, 1994; Ulusoy, 1999; von der Weth, 1999).

4. Revelation: Focusing and pattern recognition. This phase necessitates the person to prepare for the moment when a new idea finally emerges. He or she develops and enhances the idea or the product before the final stage is begun. When the basic version of the finalized representation of the task begins, one has entered the final stage (Akin \& Akin, 1998; Jones, 1992; Kristensen, 2004; O’Neill \& Shallcross, 1994).

5. Recreation: To determine full message content and express it by various methods, such as drawing. The person checks and controls the final representation for missing parts, finishes it off, and resolves it (Akin \& Akin, 1998; Jones, 1992; Kristensen, 2004; O’Neill \& Shallcross, 1994; Ulusoy, 1999).

Particular behavior patterns that define the ending of one stage and the beginning of the other are expected to occur at each stage. Among other features, the transition from readiness to reception can be most clearly identified by the beginning of observation of the task at hand with inspiration, and the one from reception to reflection is marked by the ending of observation and the beginning of externalization of ideas. Revelation is identified by increasing level of representation for the solution as a physical entity (like drawing sketches), as well as a certain amount of imagery and looking around. Finally, recreation can be recognized by the generation of idea and the finalization of details (like the beginning of rendering and effort in providing texture and materials for this particular project). At each of these stages, mental imagery and external representation takes place in different proportions, and they are significant for understanding the creative process.

\section{Mental Imagery and External Representation}

In the cognition literature, mental imagery and external representation are implicit parts of the stages of creative process (Ahsen, 1984; Bagley \& Hess, 1984; Daniels-McGhee \& Davis, 1994), and are often regarded as two equivalent means of creative problem-solving activity in design process. However, mental imagery and external representation are related to one another in an ontologically different manner. While every mental imagery process does not result in an act of external representation, external representation presupposes mental imagery. In the same way, although the formation of the creative product assumes 
the creative process (Hennessey, 1994), not every creative process leads to a creative product. Therefore, mental imagery and external representation are essential in investigations of creativity.

Creativity and imagery are very closely connected. Creativity undeniably involves imagination, that is, forming the mental image of something that does not exist. Imagery involves the formation of an individual subset of unique ideas that are involuntary and controlled sources of novel interpretations derived from a larger domain (Simonton, 2003). There is a continuous process of selection, revision, and improvement of a potentially creative idea or product (Daniels-McGhee \& Davis, 1994). The primitive thought that is externalized begins a continuous cycle of repetitions of imagery and representation until, at one point, the designer decides to draw sketches. Sketching is considered as a substantial component of creativity (Goldschmidt, 1991) and the interaction of the designers with their sketches as a creative act in design process (Akin, 1984; Rosenman \& Gero, 1998; Suwa \& Tversky, 1997; Verstijnen, Leeuwen, Goldschmidt, Hamel, \& Hennesey, 1998). Sketches quicken the process, improve results greatly, and aid the restructuring of the problem that has been given. This process is especially helpful for designers who have learned to use the sketch as a tool, and necessitates discovering new information by combining the new input and previous knowledge (Abel, 1981; Demirkan, 2005; Suwa \& Tversky, 1997; Verstijnen et al., 1998). Sketching and seeing oneself actually interacting with the reality created in the mind are significant skills for designers that aid external representation (Yokochi \& Okada, 2005).

Although many sources related to external representation in design dwell only on sketchingwhich is undoubtedly one of the most important tools a designer has - an outcome that is a result of a mental imagery process need not be in the form of a sketch. This representation can range from the writing of related keywords to a form achieved by folding paper. Consequently, sketching is only one of many different ways of externalizing thoughts in design process. These thoughts are put aside and the selection of one particular solution that is found worthy takes place as a result of several trials. This design process as a creative act should be explored within a model framework considering imagery and external representation.

\section{Framework of the Proposed Model}

Mental imagery and external representation are not only crucial in understanding the creative process, but also in developing a model to help enhance it. Moreover, the use of time in creative problem solving is a significant issue that has consequences on mental imagery and external representation in design process. A creativity model for enhancing the design studio process is developed taking into consideration these points based on the approaches and models previously discussed (Figure 1).

This model approaches the creative person, process, and product as the interactive elements within the environment. The environment is necessarily separated as the physical and social environments, and considered as the shell in which the process takes place. The 5R's of Sensational Thinking model of O'Neill and Shallcross (1994) are adapted to design process within the context of design literature, as discussed earlier. In addition, the 5R's of the model for sensational thinking played a significant role in the development of tools used in this study. This framework provides a basis for comprehensive understanding of the creative process within the design studio.

The study aimed to analyze how understanding the creative process that involves cognitive components can increase the creative quality or characteristic of the decisions made. Other

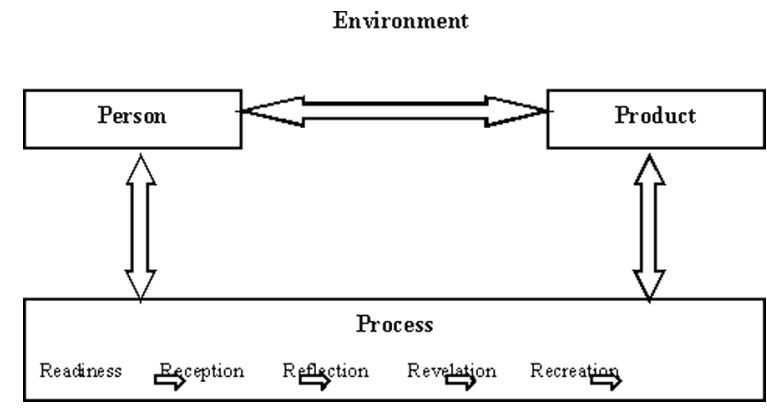

Figure 1. 
supportive issues that are analyzed within the scope of the study are as follows:

- the relationship between the observed creative traits and product creativity; the relationship between the type and quantity of representation, and creativity; and the relationship between imagery and creativity.

\section{Method}

\section{Participants}

The sample was comprised of the third-year design students of the department of Interior Architecture and Environmental Design at Bilkent University. As Dacey (1989) mentioned, creative cognition and production take place in cycles and at certain periods in life, and at the third year, the students have learned the discourse of design, formed a certain approach or style, and can clearly identify them while or after dealing with a task. These characteristics are also required in the protocol analysis method that is used in identifying the creative decisions in the design process. There were 15 subjects who were selected by random sampling among 46. Subjects were chosen by random sampling technique instead of voluntary basis because volunteers might be more motivated to complete the task, and this might have influenced the results of the study.

The group of students was comprised of 5 male and 10 female students. The mean age of the students was 20.93, with the youngest being 20 , and the oldest 23 . The means of the university entrance scores and the cumulative grade point averages (GPAs) were found to be 163.28 and 2.45 , respectively.

\section{Design Brief}

The task was to design the public area of a Theme Train and draw its perspective. The public area is the lounge or restaurant of the train that can be used by 20 people, including a 7 squaremeter service area. The plans and sections of the existing train were included within the design brief. The students had to propose their own theme, and the interior of the train should be designed accordingly. Although the students had not resolved the layout at that time, it was important for them to start to think about what kind of atmosphere they wanted to have within the train. In order to draw sketches, the students first had to form a concept that would help them in defining the theme and the atmosphere of the space. Since the students had not begun the planning of the layout of the train, this task aimed to investigate what kind of space the students had visualized prior to any kind of planning activity. As Pereira (1999) claimed, it was expected that the students would experience more flexible and creative thinking process.

Task date. The task date was particularly chosen for a number of reasons. First, it was right after the research phase of the project. Therefore, the subjects had just acquired information regarding fundamental design elements of the project. Moreover, it was claimed that significant design decisions usually were made at the beginning of a creative process (Jones, 1992; Kristensen, 2004; O’Neill \& Shallcross, 1994) and also, this was confirmed by the pilot study. It is expected that on this day, a useful concept will be found and judgments regarding the layout and atmosphere will be made. Finally, the task is to be completed within a day and the products will be available at the end of the design studio period for assessment purposes.

As the task was carried out in a single studio day, the whole process within that day was videotaped. The students began working on the task at 10:40 a.m. and continued until about 12:30 p.m., when most took their lunch-breaks. Around 1:30 p.m., most of the students were back at their tables continuing to work on the problem. The camera was on throughout the day, even when all the students were out, recording the ones when they come back to determine the length of each break. The task was due at 4:30 p.m., and all of the students submitted their drawings in time.

Setting of the study. The third year design studio for 46 students in the Department of Interior Architecture and Environmental Design was the setting for this study. The subjects were grouped in three and each was taped in detail 


\section{Hasirci and H. Demirkan}

with a different camera. In order to achieve a better idea about the relationship between the students and their use of the studio environment, a fourth camera was placed further away, taping all of the groups and the rest of the studio.

\section{Tools of Assessment}

The tools used in assessment were observation, protocol analysis, and rating scales. While observation was utilized during the creative design process, the latter two were carried out following the task (Hasirci, 2005).

Observation. The observation was done with the aid of three different assessment sheets, namely, observation of the person, process, and stages of creative problem solving. Each assessment sheet is composed of a number of components such as originality, sensitivity, or negativity. The behavioral characteristics that made up each of these components were derived from previous research and literature, and set up as oppositional phrases (Albert, 1990; Bailin, 1994; Barron, 1988; Beattie, 2000; Candy \& Edmonds, 1999; Cropley, 1999; Dorst \& Cross, 2001; Feldhusen, 1993; Guilford, 1968; Hasirci \& Demirkan, 2003; Isaksen, Puccio, \& Treffinger, 1993; O’Neill \& Shallcross, 1994). The observation of the person was carried out throughout the single day on which the task was given. The person part consists of originality, completion, self-courage, sensitivity, negativity, isolation, control, and humor components. Each component was defined by 3 to 17 particular behavioral items.

The assessment of the process was carried out on two different levels. The first one was used to determine the creativeness of the process of each student. This part of the process consists of originality, completion, self-courage, sensitivity, negativity, identification, and movement components. Each component was defined by two to ten particular behavioral items. The second process assessment was used to determine the stages of creative problem solving and the duration of each stage. The behavioral characteristics that determine each stage were derived from previous research and literature (Bailin, 1994; Beattie, 2000; Candy \& Edmonds, 1999; Cropley, 1999;
Dacey, 1989; Dorst \& Cross, 2001; Feldhusen, 1993; Guilford, 1968; Isaksen, Puccio, et al., 1993; O'Neill \& Shallcross, 1994). Certain behavior patterns are expected to occur at each stage, which also define the ending of one stage and the beginning of the other. Each stage was defined by 6 to 13 particular behavioral items.

The understanding of the stages were supported by means of videotaping the whole experiment. This technique aided in obtaining any extra information that may be helpful to the study. The individual videotaped sessions were then watched with each student to discuss and double check the observer's interpretation of the student's work and actions. The internal consistency of the items in each observation sheet were assessed with Cronbach's alpha (Cronbach, 1951), and only those with alphas over .80 were considered.

\section{Protocol Analysis}

Either think-aloud or the retrospective protocol analysis method is reliable and common in the analysis of cognitive processes (Ericsson \& Simon, 1993). Due to the disadvantage of interfering with the design process, the think-aloud method was excluded. As supporting the retrospective method, Dorst \& Cross (2001) stated that one can more easily identify the moment that the crucial and focal concept began to emerge after the completion of the process. The subjects were asked 13 open-ended questions related to the stages of design process, methods, and techniques used in problem solving, and their thoughts about being more creative. As it is difficult to understand the nature of imagery process with only observation, the protocol analysis system was also used. Retrospective protocol analysis is considered a very reliable method and used extensively in the investigation of imagery in various fields (Brain, Haines, \& Williams, 1998; Dahl, Chattopadhyay, \& Gorn, 1998; Eastman, Newstetter, \& McCracken, 2001; Finke, 1996; Finke, Ward, \& Smith 1992; Ritz, Alatrapa, Thons, \& Dahme, 2002).

\section{Rating Scales}

The product part consists of the individual assessments of the components of product 
creativity, design elements, unifying principles, and spatial qualities. The definition of each factor under these titles was given to the instructors to ensure clearance on it.

The assessment of the product was done according to the following factors:

1. Characteristics of creativity: value, appropriateness, flexibility, fluency, novelty, originality, elaboration, redefinition, ability to answer needs, and open-endedness (evolution).

2. Design elements: line, shape and form, space, texture, value, color, and light.

3. Unifying principles: repetition, variety, rhythm, balance, emphasis, unity, and harmony.

4. Spatial qualities: concept execution, atmosphere/ ambiance (material, color, texture, lighting), planning/layout, building system and components (heating, ventilation and air-conditioning, sound system), ergonomics (health, safety, comfort), use of standards, furniture (choice, design, utilization), design details, material use, presentation, and craftsmanship.

Instead of oppositional phrases, the assessment was done on a five-point scale (poor, poor-average, average, average-excellent, excellent) as the product characteristics necessitate a more detailed categorization. The instructors and the observer assessed each student's work independently; thus, the possibility of affecting each other while grading the products were eliminated. The raw averages of the two scores were calculated for the final performance score, and a paired sample $t$-test was carried out to assess the difference between the instructor's and the observer's rating on the product. In total, no significant difference was found between the assessments $(t=-1.00$, $d f=14, p>0.05$ ).

\section{Results}

\section{Overall Results}

The mean scores of the person and process of the subjects were calculated one by one, taking the overall average of all the behavioral characteristics that compose each component of the related observation sheets. The mean of the product was calculated by taking the average of the four factors that compose this element in the rating scales. Then it was assumed that person, process, and product have equal weights and each value was standardized (see Table 1).

The product moment correlation between the three elements of creativity, university entrance exam score, and GPAs was analyzed (see Table 2). The university entrance exam scores and the GPAs of the students were highly correlated $(r=.84)$. There was a rather low correlation between entrance exam scores and person $(r=.60)$ and GPA and overall creativity $(r=.52)$. Naturally, these scores of students were not correlated with the creativity scores regarding the process and product. The university entrance exam scores are obtained by the number of correctly answered questions based on the previously learned material; the GPA is the total score derived from a number of various courses that the student has taken.

When the correlation between the three elements of creativity and overall creativity was analyzed, it is found that the highest correlation was

Table 1. The Mean Scores in Percentages for Each Student Related to the Association Between the Elements of Creativity and Observation Time at the Beginning of the Task

\begin{tabular}{rrrrrr}
\hline \multicolumn{5}{c}{ Elements of Creativity (\%) } & \\
\cline { 3 - 4 } & & Person & Process & Product & \\
\hline 1 & 10 & 5.62 & 5.79 & 6.66 & 18.07 \\
2 & 45 & 17.54 & 16.39 & 18.47 & 52.40 \\
3 & 20 & 5.31 & 8.80 & 25.71 & 39.82 \\
4 & 35 & 18.63 & 11.02 & 24.42 & 54.07 \\
5 & 35 & 18.53 & 21.56 & 13.71 & 53.80 \\
6 & 20 & 9.20 & 4.76 & 6.66 & 20.62 \\
7 & 20 & 21.86 & 19.04 & 6.66 & 47.56 \\
8 & 10 & 7.02 & 1.90 & 7.05 & 15.97 \\
9 & 15 & 16.85 & 1.90 & 9.33 & 28.08 \\
10 & 20 & 20.89 & 20.33 & 12.76 & 53.98 \\
11 & 30 & 13.13 & 4.76 & 9.33 & 27.22 \\
12 & 15 & 12.34 & 17.58 & 18.66 & 48.58 \\
13 & 15 & 16.75 & 5.39 & 6.66 & 28.80 \\
14 & 15 & 16.58 & 13.33 & 6.85 & 36.76 \\
15 & 20 & 15.03 & 11.90 & 13.71 & 40.64 \\
\hline
\end{tabular}

Note. Two decimals have been furthered in each percentage. The internal validities of the observational features were controlled by Alpha Cronbach tests, and the only the validities over 0.8 were taken into consideration. 


\section{Hasirci and H. Demirkan}

Table 2. Correlation Among Three Elements of Creativity

\begin{tabular}{|c|c|c|c|c|c|c|c|c|c|}
\hline & & & & & \multicolumn{5}{|c|}{ Product } \\
\hline & & & & & $\begin{array}{l}\text { Product } \\
\text { Creativity }\end{array}$ & $\begin{array}{c}\text { Design } \\
\text { Elements }\end{array}$ & $\begin{array}{l}\text { Unifying } \\
\text { Principles }\end{array}$ & $\begin{array}{c}\text { Spatial } \\
\text { Qualities }\end{array}$ & $\begin{array}{l}\text { Product } \\
\text { (Mean) }\end{array}$ \\
\hline GPA & $0.835^{*}$ & & & & & & & & \\
\hline Person & $0.604^{*}$ & 0.488 & & & & & & & \\
\hline Process & 0.333 & 0.421 & $0.606^{*}$ & & & & & & \\
\hline Product creativity & 0.046 & 0.293 & -0.028 & 0.403 & & & & & \\
\hline Design elements & -0.060 & 0.195 & -0.035 & 0.345 & $0.882^{*}$ & & & & \\
\hline Unifying principles & -0.064 & 0.097 & -0.023 & 0.231 & $0.848^{*}$ & $0.947^{*}$ & & & \\
\hline Spatial qualities & -0.100 & 0.163 & -0.154 & 0.264 & $0.867^{*}$ & $0.953^{*}$ & $0.937^{*}$ & & \\
\hline Product (mean) & 0.033 & 0.258 & -0.015 & 0.314 & $0.961^{*}$ & $0.938^{*}$ & $0.938^{*}$ & $0.923^{*}$ & \\
\hline Overall mean & 0.416 & $0.522^{*}$ & $0.681^{*}$ & $0.880^{*}$ & $0.648^{*}$ & $0.605^{*}$ & $0.554^{*}$ & 0.512 & $0.628^{*}$ \\
\hline
\end{tabular}

Note. ${ }^{*}$ Correlation is significant at the 0.05 level (2-tailed).

between process and overall creativity $(r=.88)$. Then it was followed by person $(r=.68)$ and product $(r=.63)$, respectively.

One-way analysis of variance showed that there was no significant difference between the three elements of creativity $(F=2.552, d f=2, p=0.079)$. Multiple regression was used to estimate how well the subjects would achieve in a product design if they had certain creativity characteristics. The regression equation was Product $=12.725$ 0.399 (Person) +0.496 (Process). The creativity of the person and process have value in forecasting who will succeed in product creativity (Hasirci \& Demirkan, 2003).

\section{Results Related to Time Usage in Stages of Design Process}

Based on the second observation sheet related to process and protocol analysis, the time spent at each stage by each student is depicted in Figure 2. On average, $48 \mathrm{~min}$ were spent in the readiness stage, 81 in the reception stage, 57 in the reflection stage, 55 in the revelation stage, and 35 in the final, recreation, stage. The students usually spent more time in the reception stage, compared to the final stage of recreation, which means that they did not have time to finalize their perspective drawings and prepare them for assessment. Only two students (subjects 4 and 5) could come closer to finishing their perspective drawings, and they were among the few who had spent more time (75 and $60 \mathrm{~min}$, respectively) on the final stage of recreation compared to the previous stages. However, these two students had also spent a considerable amount of time ( $45 \mathrm{~min}$ each) during their readiness stage gazing around in a daydreaming-like mode, which suggested their use of imagery at that time.

\section{Results Related to Retrospective Protocol Analysis}

The findings derived from the retrospective interviews are discussed under the headings of Tools and roblem-solving methods, Inspiration sources, and Use of time and creativity.

Tools and problem-solving methods. Among 15 students, 7 stated that they solved the design problem using $70 \%$ imagery and $30 \%$ representation. Following this group, 5 students stated that they used imagery and representation equally (50\% each) throughout the design process. Two students said that they used more than $70 \%$ imagery and less than $30 \%$ representation, and only one student said that he used 30\% imagery and 70\% representation. All of the students stated that the distribution of representation and imagery usage and representation techniques were consistent in all projects.

Two-dimensional representations were used as a common tool in visualization of the three-dimensional space. Different two-dimensional representation techniques were named by students according to the given problem domain. It is also likely that they are missing the importance of the 


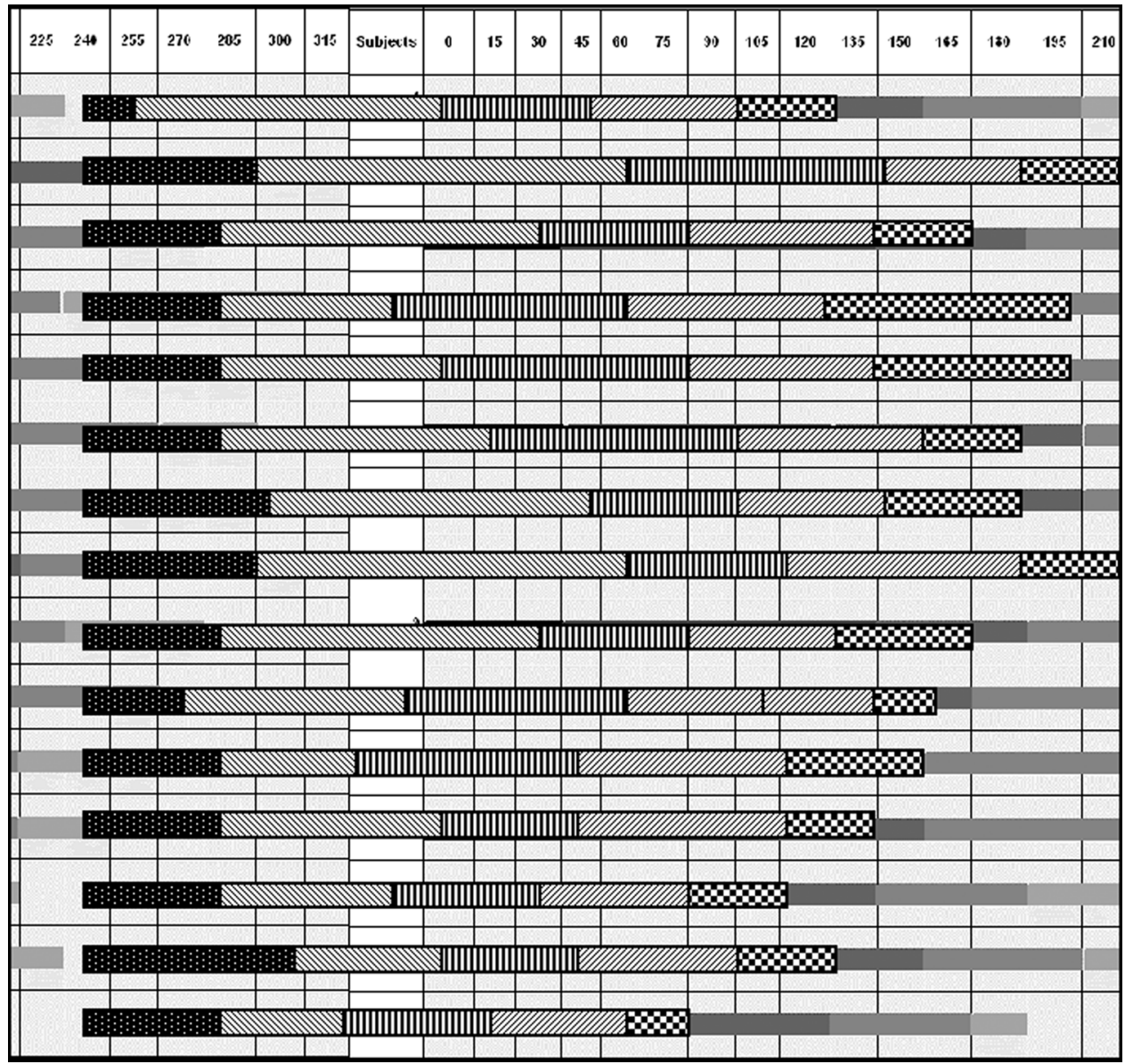

Figure 2. Duration of the five stages of O'Neill and Shallcross's (1994) Sensational Thinking model (Gantt Chart).

skill of sketching, which they can carry out anywhere and will be quite helpful in their professional lives, as a means of representation, as well as creative problem solving. Although it is becoming simpler to use computer programs each day, it is important for a designer to develop the skill of sketching to be able to demonstrate onthe-spot ideas and solutions to clients, collaborators, and workers. Representation in color was mentioned as a useful method of imagining the ideas. However, according to the rating of instructors, the use of color in their projects was not well developed. Because use of color aids delivering ideas on texture and atmosphere as well, this is an important skill that should be developed and stressed in the curriculum.
Only three students mentioned models or 3-dimensional objects as an aid to develop the creative process. Among other ways to facilitate the problem-solving process, computerized drawings were mentioned by two students as it was easy to draw on computers, see the project details, and make changes on them as necessary. The majority of the students mentioned spoken words or oral representation as a creative tool. This shows that students use imagery more than the representation of their ideas.

Inspiration sources. Design magazines, books, and the Internet were frequently mentioned inspiration sources by the students. Moreover, all students stressed the impact of critiques on 
effective reasoning for turning points and milestones of the design process. Beside these sources, specific issues related to the task (designing a theme train) were among the common answers such as design details of the vehicle and lighting system, chosen colors, themes, and concepts. Also, the issue of creating a maximally equipped space out of a constrained one was seen as a challenge that forced the students to push their creative limits.

The students mentioned that they felt competent as interior architects, as they were overcoming the various problems that an average person cannot solve throughout the design process. Although the students repeatedly stated that, they did not feel competent in representing their ideas on paper due to an inhibition related to sketching and thinking by sketching. Besides the stated inspiration sources like design books, design magazines, and the Internet, these were mentioned as being effective by giving rise to radical changes in the normal flow of the creative process.

Use of time and creativity. Regarding the time of the most creative idea, the students believed that it was the end of the day when they worked on finalizing their drawings. This has also been identified as a time at which utmost representation and minimum imagery takes place, as can be observed from the videotapes. Also, juries and project due times were stated as being effective on creativity. Specifically, three students stated that as they get closer to running out of time, they have the most creative ideas that they had not thought about before. This finding is consistent with the stages of creative problem solving - the 5R's-which explains that revelation and recreation take place close to the finalization of the process (O'Neill \& Shallcross, 1994). The selection of the theme or concept is one of the first steps that one takes before elaborating on the process, and is one that also resulted in high levels of creativity according to the students.

The issues that make the students work more efficiently were mentioned as: inspirational ideas, an environment without interruptions, more time and less pressure of deadlines, research, critiques, television, design magazines, a warmer environment with less pressure that is nevertheless disciplined, and drawing more sketches. A recurring issue that came up in the interviews was that the students found their decisions regarding the color scheme to be the most creative decision they had throughout the process.

The responses regarding the issues related to the most time spent were quite consistent. Following the initial stage in which the students are first introduced to the project, they were expected to choose a theme or concept to guide them through the process and reflect their opinions on what the general atmosphere of the environment should be like. Afterwards, the students concentrated on reflecting this theme onto the project, which was mentioned to be the stage at which the students spent the most time. This was also identified as a stage at which utmost imagery and minimum representation took place, as it is observed from the videotapes. The act of drawing a perspective has also been stated to be very difficult, as most of the students had a hard time remembering the rules and drawing one without distortion. Twelve students stated that they spent the most time during the planning phase on the perspective drawings.

Regarding the responses on the issues that would make them work more creatively, the students mentioned issues that were not that different than the things that would make them more efficient. Among the responses were having more time, doing group-work, less pressure of deadlines, preparing more drawings and sketches, more critiques, more research, a friendlier environment with music, and more exposure to design magazines and books.

There was no significant relationship between observation time and creativity level during design process (two-tailed Fischer exact $p=.60$ ). Although the student who has received the lowest total creativity score has spent the least observation time $(10 \mathrm{~min})$, and the student who has received the highest score has spent a considerable amount of time $(35 \mathrm{~min})$, the duration of observation time is not dependent on creativity level.

\section{Discussion}

The findings of the study are discussed according to the four stated issues within the framework 
of the proposed model: how understanding the creative process that involves cognitive components can increase the creative quality/characteriacteristic of the decisions made. Putting the creative process under the microscope was helpful in understanding preferred imagery and representation styles and quantity, time spent at different stages of the process, underdeveloped skills, and behavior, in addition to student-student and student-instructor relations, and associations between creative processes and products. Understanding these relations can be helpful in developing the curriculum to aid the skills necessary to experience more creative processes conducive to produce more creative products in the design studio. The results can then be extended into practice.

\section{The Relationship Between the Observed Creative Traits and Product Creativity}

This relationship can easily be seen in the observations. Those students who did not only work on their projects, but also spent time on asking others' opinions and discussing their projects were among the most creative ones. This, of course, should not be confused with mingling with everyone in the studio and killing time. A constructive interaction between the students was observed to be helpful in developing their ideas. During these exchanges of ideas, they had the opportunity of noticing something they had not noticed before, see different ways of looking at the problem, or develop an existing idea. Even visual contact has been observed to be useful. That is, seeing another student working and seeing what he or she is doing was observed to motivate the students and keep up with the same phase. The students in each group completed the phases with similar timing and submitted the project almost simultaneously, making the three groups consistent within the groups in terms of timing, although most seemed to prefer being alone for the first creative idea. The arrangement of the studio environment affected the behavior of the students, and three students mentioned becoming more motivated when working individually in a group arrangement of tables. Simonton (2003) agreed that working within a larger group of people on different levels enables the interaction of creative ideas as a result of being subject to the work of classmates and predecessors of the field.

\section{The Relationship Between the Type and Quantity of Representation, and Creativity}

Three-dimensional representations such as perspective drawings and models were found to lead to more creativity, compared to two-dimensional depictions such as layouts and plans. Crosssections were nevertheless mentioned, as they enable one to see various features inside the space on different levels. Thus, more importance should be given to sketching, so that this significant skill of designers can fully develop in a way that enables the students to easily represent what they visualize in their minds. The skill of visualizing and representing the visualized necessitates more attention and exercise on the part of the students. Finally, while verbalization during the design process and among classmates can be constructive, as also supported by Eastman et al. (2001), excessive reliance on verbalization in critiques appears to weaken the skill of sketching.

\section{The Relationship Between Imagery and Representation Amounts and Creativity}

There was no significant relationship between imagery amount and creativity level during design process (two-tailed Fischer exact $p=0.622$ ). Two of the most creative students used $80 \%$ imagery (highest percentage), and one of the least creative students used 30\% imagery (lowest percentage). Because imagery was generally used in the beginning phases, it can be stated that the initial ideas and concepts were found mostly by this technique. However, imagery alone cannot be sufficient to improve an idea, and good representational skills are crucial in the development phases.

The students who made use of their readiness stage by utilizing imagery were found to be the more creative ones. However, these students were also the ones who actually did use this time to their advantage instead of wasting their time walking around the studio or as an extended prereadiness stage in which the students prepare the equipment that they plan to use during the process. This results in an ineffective use of time and necessitates the students to 


\section{Hasirci and H. Demirkan}

jump from the prereadiness stage to the reception stage without fully living the previous. The students who skip this stage also miss out on certain important uses of this stage, such as visualizing the space they will be creating in their minds.

\section{Conclusion}

This study investigated the creative person, process, and product as the essential components of creativity within a design studio environment. Results showed that the highest correlation was between process and overall creativity. Also, the issues that supported creative process were found to be keeping the goals of the task explicit from the very beginning, helping students become aware of their own creative problem-solving methods, effective time usage, having short series of imagery exercises in concept development, visualizing the space before drawing the layout, and encouraging students to identify themselves with the users. Furthermore, group work, inspiring students to personalize their studios, and making them aware of criticism techniques about both their own work and their classmates may also enrich the creative process and the products that come out as a result of that. The model developed in this study can be utilized to create an atmosphere in which students can work in a way to enhance creative potential, bring about suggestions for curriculum changes, and improve designs of the students both in the studio and after graduation.

\section{References}

Abel, C. (1981). Function of tacit knowing in learning to design. Design Studies, 2, 209-214.

Ahsen, A. (1984). ISM: The triple code model for imagery and psychophysiology. Journal of Mental Imagery, 8, 15-42.

Akin, O. (1984). Psychology of architectural design. Pittsburgh, PA: Carnegie-Mellon University.

Akin, O., \& Akin, C. (1996). Frames of reference in architectural design: Analysing the hyperacclamation (A-h-a-!). Design Studies, 17, 341-361.

Akin, O., \& Akin, C. (1998). On the process of creativity in puzzles, inventions, and designs. Automation in Construction, 7, 123-138

Albert, R. S. (1990). Identity, experiences, and career choice among the exceptionally gifted and talented. In
M. A. Runco \& R. S. Albert (Eds.), Theories of creativity (pp. 13-34). Newbury Park, CA: Sage.

Bagley, M. T., \& Hess, K. K. (1984). Imagery in the classroom. New York: Trillium.

Bailin, S. (1994). Achieving extraordinary ends: An essay on creativity. Norwood, NJ: Ablex.

Bandrowski, J. F. (1985). Creative planning throughout the organization. New York: American Management Association.

Barron, F. (1988). Putting creativity to work. In R. J. Sternberg (Ed.), The nature of creativity (XX-XX). Cambridge, England: Cambridge University Press.

Beattie, D. K. (2000). Creativity in art: The feasibility of assessing current conceptions in the school context. Assessment in Education, 7, 175-192.

Brain, K. L., Haines, J., \& Williams, C. L. (1998). The psychophysiology of self-mutilation: Evidence of tension reduction. Archives of Suicide Research, 4, 227-242.

Candy, L., \& Edmonds, E. (1996). Creative design of the Lotus bicycle: Implications for knowledge support systems research. Design Studies, 17, 71-90.

Candy, L., \& Edmonds, E. (1999). Introducing creativity to cognition. Loughborough, UK: ACM.

Christiaans, H. H. C. M. (1992). Creativity in design: The role of domain knowledge in designing. Utrecht, XX: Lemma.

Christiaans, H. H. C. M. (2002). Creativity as a design criterion. Creativity Research Journal, 14, 41-54.

Cronbach, L. (1951). Coefficient alpha and the internal structure of tests. Psychometrika, 12, 1-16.

Cropley, A. J. (1999). Creativity and cognition: Producing effective novelty. Roeper Review, 21, 253-261.

Cross, N. (1997). Descriptive models of creative design: Application to an example. Design Studies, 18, 427-455.

Cross, N. (2002). Creative cognition in design: Processes of exceptional designers. In T. Hewett \& T. Kavanagh (Eds.), Creativity and cognition (pp. 14-19). New York: ACM Press.

Dacey, J. S. (1989). Fundamentals of creative thinking. Lexington, MA: D. C. Health.

Dahl, D. W., Chattopadhyay, A., \& Gorn, G. J. (1998). Improving the new product design process: The role of visual mental imagery. In Proceedings of ANZMAL (pp. 320-331). New Zealand: Duneidin.

Daniels-McGhee, S., \& Davis, G. A. (1994). The imagerycreativity connection. The Journal of Creative Behavior, 28, 151-177.

Demirkan, H. (2005). Generating design activities through sketches in multi-agent systems. Automation in Construction, 14, 699-706.

Dorst, K., \& Cross, N. (2001). Creativity in the design process: Co-evolution of problem and solution. Design Studies, 22, 425-437.

Eastman, C., Newstetter, W., \& McCracken, M. (Eds). (2001). Design knowing and learning: Cognition in design education. Amsterdam: Elsevier Science.

Ericsson, K. A., \& Simon, H. A. (1993). Protocol analysis: Verbal reports as data. Cambridge, MA: MIT Press.

Feldhusen, J. F. (1993). A conception of creative thinking and creativity training. In S. G. Isaksen, M. C. Murdock, R. L. Firestien, \& D. J. Treffinger (Eds.), Nurturing and 
developing creativity: The emergence of a discipline (pp. 31-50). Norwood, NJ: Ablex.

Finke, R. A. (1996). Imagery, creativity, and emergent structure. Consciousness and Cognition, 5, 381-393.

Finke, R. A., Ward, T. B., \& Smith, S. M. (1992). Creative cognition. Boston: MIT Press.

Fritz, R. (1991). Creating. New York: Fawcett.

Gero, J. S. (1994). Computational models of creative design processes. In T. Dartnall (Ed.), Artificial intelligence and creativity (pp. XX-XX). Dordrecht, The Netherlands: Kluwer Academic.

Goldschmidt, G. (1991). The dialectics of sketching. Creativity Research Journal, 4, 123-143.

Guilford, J. P. (1968). Intelligence, creativity, and their educational implications. San Diego, CA: Robert R. Knapp.

Hasirci, D. (2005). Understanding the effects of cognition in creative decision-making: A creativity model for enhancing the design studio process. Ankara, Turkey: Unpublished doctoral dissertation, Bilkent University.

Hasirci, D., \& Demirkan, H. (2003). Creativity in learning environments: The case of two sixth grade art-rooms. Journal of Creative Behavior, 37, 17-42.

Hennessey, B. A. (1994). The consensual assessment technique: An examination of the relationship between ratings of product and process creativity. Creativity Research Journal, 7, 193-208.

Isaksen, S. G., \& Treffinger, D. J. (1985). Creative problem solving: The basic course. Buffalo, NY: Bearly.

Isaksen, S. G., Murdock, M. C., Firestien, R. L., \& Treffinger, D. J. (Eds.) (1993). Nurturing and developing creativity: The emergence of a discipline (pp. 1-7). Norwood, NJ: Ablex.

Isaksen, S. G., Puccio, G. J., \& Treffinger, D. J. (1993). An ecological approach to creativity research: Profiling for creative problem solving. Journal of Creative Behavior, 27, 149-170.

Jones, J. C. (1992). Design methods. New York: Van Nostrand Reinhold.

Jones, L. (1993). Barriers to creativity and their relationship to individual, group, and organizational behavior. In S. G. Isaksen, M. C. Murdock, R. L. Firestien, \& D. J. Treffinger (Eds.), Nurturing and developing creativity: The emergence of a discipline (pp. 133-154). Norwood, NJ: Ablex.

Koberg, D., \& Bagnall, J. (1981). The all new universal traveller: A soft-systems guide to creativity, problem-solving, and the process of reaching goals. Los Altos, CA: William Kaufmann.

Kristensen, T. (2004). The physical context of creativity. Creativity and Innovation Management 13, 89-96.

Krueger, C., \& Cross, N. (2001). Modelling cognitive strategies in creative design. In J. Gero \& M. Maher (Eds.), Computational and cognitive models of creative design $V$ (pp. 1-25). Sydney, Australia: University of Sydney.

Lawson, B. (1994). Design in mind. Oxford, UK: Butterworth Architecture.

Lloyd, P., \& Snelders, D. (2003).What was Philippe Starck thinking of? Design Studies, 24, 237-253.

Mooney, R. L. (1963). A conceptual model for integrating four approaches to the identification of creative talent. In
C. W. Taylor \& F. Barron (Eds.), Scientific creativity: Its recognition and development (pp. 331-340). New York: Wiley.

O’Neill, S., \& Shallcross, D. (1994). Sensational thinking: A teaching/learning model for creativity. The Journal of Creative Behavior, 28, 75-88.

Osborn, A. (1953) Applied imagination. New York: Charles Scribner.

Pereira, L. Q. (1999). Divergent thinking and the design process. In P. H. Roberts \& E. W. L. Norman (Eds.), Proceedings of the international conference on design and technology educational research and curriculum development (pp. 224-229). Loughborough, England: Loughborough University, Department of Design and Technology.

Plsek, P. E. (1997). Working paper: Models for the creative process. Retrieved June 15, 2003, from http://directedcreativity. com/pages/WPModels.html

Ritz, T., Alatrapa, S., Thons, M., \& Dahme, B. (2002). Effects of affective picture viewing and imagery on respiratory resistance in nonasthmatic individuals. Psychophysiology, 39, 86-94.

Rhodes, M. (1987). An analysis of creativity. In S. G. Isaksen (Ed.), Frontiers of creativity research: Beyond the basics (pp. 216-222). Buffalo, NY: Bearly. (Original work published 1961)

Rosenman, M. A., \& Gero, J. (1993). Creativity in design using a design prototype approach. In J. Gero \& M. L. Maher (Eds.), Modelling creativity and knowledge-based creative design (pp. XX-XX). XXCITY, NJ: Lawrence Erlbaum Associates, Inc.

Rosenman, M. A., \& Gero, J. (1998). Purpose and function in design. Design Studies, 19, 161-186.

Rossman, J. (1931). The psychology of the inventor. Washington DC: Inventor's Publishing.

Roy, R., \& Design Innovation Group. (1998). Case studies of creativity in innovative product development. Design Studies, 14, 423-443.

Runco, M. A. (2004). Creativity. Annual Review of Psychology, $55,657-687$.

Simonton, D. K. (2003). Scientific creativity as constrained stochastic behavior: The integration of product, person, and process perspectives. Psychological Bulletin, 129, 475-494.

Suwa, M., \& Tversky, B. (1997). What do architects and students perceive in their design sketches? A protocol analysis. Design Studies, 18, 385-403.

Ulusoy, Z. (1999). To design versus to understand design: The role of graphic representations and visual expressions. Design Studies, 20, 123-130.

Verstijnen, I. M., Leeuwen, G., Goldschmidt, G., Hamel, R., \& Hennesey, J. M. (1998). Sketching and creative discovery. Design Studies, 19, 519-546.

von der Weth, R. (1999). Design instinct?-The development of individual strategies. Design Studies, 20, 453-463.

Wallas, G. (1926). The art of thought. New York: Harcourt Brace Jovanovich.

Yokochi, S., \& Okada, T. (2005). Creative cognitive process of art making: A field study of a traditional Chinese ink painter. Creativity Research Journal, 17, 241-257 